\section{ROBOTIC INTERVAL DEBULKING SURGERY FOR ADVANCED EPITHELIAL OVARIAN CANCER. CURRENT CHALLENGE OR FUTURE DIRECTION? A SYSTEMATIC REVIEW}

Victoria Psomiadou, Anastasia Prodromidou, Alexandros Fotiou, Sofia Lekka, Christos R lavazzo. Metaxa Cancer Hospital, Piraeus, Greece; Metaxa Memorial Cancer Hospital; Department of Gynecologic Oncology

\subsection{6/ijgc-2020-ESGO.150}

Introduction/Background Safety and efficacy of robotic interval debulking surgery (IDS) after treatment with neoadjuvant chemotherapy (NACT) in advanced epithelial ovarian cancer (EOC) was evaluated.

Methodology A systematic review of the literature was conducted.

Results We evaluated 102 patients in total. Perioperative outcomes were estimated as following: mean estimated blood loss ranged from 106.9 to $262.5 \mathrm{ml}$ (mean $\pm \mathrm{SD}$ : $168 \pm 68 \mathrm{ml}$ ), mean operative time ranged from 164 to 312 min (mean \pm SD: $246 \pm 61 \mathrm{~min})$, mean hospital stay was 2.4 days and postoperative blood transfusion rate was $19 \%(n=19 / 98)$. Regarding the oncological outcomes, 75 patients received a R0 resection (complete cytoreduction), while by 21 women there was a residual disease $\leq 1 \mathrm{~cm}$. Regarding complications, no intraoperative and 6 postoperative $(14,6 \%)$ complications were recorded, with a 30-d mortality rate of 9.2\% $(n=9 / 98)$, whereas the laparotomy conversion rate was $9.2 \%(9 / 98)$ as well, mostly in the terms of achieving complete cytoreduction. During a median follow up period of 2 to 86 months (median 25.3 months), the median overall survival from 39.7 to 47.2 months and the progression free survival varied from 20.6 to 21.2 months. Recurrent disease was reported in 60 women $(61 \%)$. Our results are in harmony $(p=0.02)$ with those of the one study that presented significantly improved OS and PFS in the robotic arm compared to laparotomy (47.2 vs 37.8 vs $37.9, p=0.004$ for OS and 20.6 vs 13.9 vs $11.9, \mathrm{p}=0.005$ for PFS, respectively).

Conclusion Robotic interval debulking surgery is a safe and efficient regarding the management of advanced ovarian cancer patients who receive neoadjuvant chemotherapy. The patients that are more eligible and could benefit from this treatment strategy should be specified through larger, doubleblind randomized control trials.

Disclosures All authors declare no conflict of interest.

\section{PERITONEAL CANCER INDEX (PCI) AS A PREDICTOR OF COMPLETENESS OF CYTOREDUCTION AT PRIMARY AND INTERVAL DEBULKING SURGERY IN ADVANCED OVARIAN CANCER}

${ }^{1}$ Paula Fagan, ${ }^{2}$ Susana Banerjee, ${ }^{3}$ Desmond Barton, ${ }^{4}$ Angela George, ${ }^{3}$ Thomas Ind, ${ }^{3}$ Marielle Nobbenhuis, ${ }^{1} J o h n$ Butler. ${ }^{1}$ The Royal Marsden Hospital; Gynaecological Oncology; ${ }^{2}$ Royal Marsden Hospital; The Royal Marsden Hospital; Department of Medical Oncology; ${ }^{3}$ The Royal Marsden Hospital; ${ }^{4}$ The Royal Marsden Hospital; Chelsea; Department of Medical Oncology

\subsection{6/ijgc-2020-ESG0.151}

Background The completeness of surgical cytoreduction is the most important prognostic factor in advanced epithelial ovarian cancer (AOC). The FIGO staging system for ovarian cancer does not accurately account for disease distribution and tumour burden within the peritoneal cavity.

The peritoneal cancer index (PCI) quantitatively assesses cancer distribution and tumour burden in the peritoneal cavity in 13 abdominopelvic regions. It does not, however, include retroperitoneal nodal disease. First described by Sugarbaker, it was widely used in colorectal cancer and peritoneal mesothelioma. More recently, the PCI has been used to quantify tumour burden in patients with AOC. It may be a suitable tool to predict the completeness of cytoreduction at primary and interval debulking surgery. The aim of this study was to analyse the prognostic value and clinical correlations of PCI in patients with AOC.

Methodology We evaluated the correlation between PCI and cytoreductive score (GOG-score) in patients with AOC who were treated with primary and interval debulking surgery at a UK tertiary cancer centre. Data for 36 consecutive patients with AOC were collected prospectively from January to September 2020. An Ovarian Cancer Reporting Tool was developed according to the ESGO Ovarian Cancer Surgery Guideline and the Dutch Hyperthermic Intraperitoneal Chemotherapy Protocol. Intra-operative PCI scores prior to and after resection were calculated using the report sheet, intraoperative findings and surgical notes. The scores were correlated to completeness of cytoreduction according to the GOGscore $(1=$ no macroscopic residual disease, $2=0.1-1 \mathrm{~cm}$ residual, $3=1-2 \mathrm{~cm}$ residual disease, $4 \geq 2 \mathrm{~cm}$ residual disease).

Results Of the 36 patients, 25\% (9/36) were staged FIGO IIIB, 33.3\% (12/36) were FIGO IIIC and 41.6\% (15/36) were FIGO IV. Twenty-five percent (9/36) underwent primary debulking surgery and $75 \%$ (27/36) underwent interval debulking surgery after neoadjuvant chemotherapy. Thirty-one $(86 \%)$ patients had high grade serous histology and five (14\%) low grade serous carcinoma. Table 1 illustrates the distribution of intra-operative PCI scores and completeness of cytoreduction.

Twenty-three (64\%) patients had a PCI of 0 to 15 . In 22 (96\%) complete cytoreduction (GOG-1) was achieved and in 1 (4\%) there was $0.1-1 \mathrm{~cm}$ residual disease (GOG-2). Four patients had a PCI of 16 to 20 with GOG-1 achieved in 3 $(75 \%)$ and GOG-2 in 1 (25\%).

Nine patients had a PCI greater than 20 and rates of cytoreduction were: GOG-1 = 4 (44\%), GOG-2 = 2 (22\%), GOG-3 = $2(22 \%)$ and GOG-4 = $1(11 \%)$.

Conclusion PCI is a reproducible and objective tool for assessing the likelihood of complete resectability at primary and interval debulking surgery for AOC. A PCI of $0-20$ was

Abstract 557 Table 1 Intra-operative $\mathrm{PCl}$ score and maximum diameter of residual disease

\begin{tabular}{lccccc}
\hline $\begin{array}{l}\text { Intra-Op } \\
\text { PCI }\end{array}$ & $\mathrm{n}=36$ & GOG- & GOG- & GOG- & GOG- \\
\hline 0 & & 1 & 2 & 3 & 4 \\
$1-5$ & 1 & 1 & & & \\
$6-10$ & 5 & 5 & & & \\
$11-15$ & 9 & 9 & & & \\
$16-20$ & 8 & 7 & 1 & & \\
$>20$ & 4 & 3 & 1 & & 1 \\
\hline
\end{tabular}

